\title{
CLOSURE OPERATORS AND PROJECTIONS ON INVOLUTION POSETS
}

\author{
GOTTFRIED T. RÜTTIMANN \\ (Received 1 May 1972; revised 30 April 1973) \\ Communicated by P. D. Finch
}

\section{Introduction}

Investigations of closure operators on an involution poset $T$ lead to a certain type of closure operators (so called $c$-closure operators) that are closely related to projections on $T$.

In terms of these operators we give a necessary and sufficient condition for an involution poset to be an orthomodular lattice. An involution poset is an orthomodular lattice if and only if it admits certain $c$-closure operators. In that case, if $L$ is an orthomodular lattice, the set of $c$-closure operators, under the usual ordering of closure operators, is orderisomorphic to the set of projections of the Baer *-semigroup $B(L)$ of hemimorphisms on $L[4]$. In this sense, but working on the "opposite end", this treatment enlarges that given in [3] where a similar necessary and sufficient condition is represented but for orthocomplemented posets and for mappings which in the case of an orthomodular lattice are exactly the closed projections of $B(L)$. $C$-closure operators appear as a natural generalization of symmetric closure operators [5].

\section{C-closure operators}

An involution poset $T$ is a poset with largest element (1) and a mapping $e \in T \rightarrow e^{\prime} \in T$ such that $e^{\prime \prime}=e$ and $e \leqq f \Rightarrow f^{\prime} \leqq e^{\prime}$. For basic definitions see $[1,2]$.

A projection $\phi$ on an involution poset $T$ is a mapping $\phi: T \rightarrow T$ with the following properties:

i) $e \leqq f \Rightarrow e \phi \leqq f \phi$,

ii) $(e \phi) \phi=e \phi$,

iii) $(e \phi)^{\prime} \phi \leqq e^{\prime} \quad(e, f \in T)$.

Work supported by The Canada Council. 
The set of projections on $T$, denoted by $P(T)$, is not empty since $I$ defined by $e I:=e$ is a projection.

LEMMA 1. Let $\phi$ be a projection on $T$. Then $\left((e \phi)^{\prime} \phi\right)^{\prime} \phi=e \phi$ is valid for all $e \in T$.

Proor. Since $(e \phi)^{\prime} \leqq e^{\prime}$ for all $e \in T$, it follows that $\left((e \phi)^{\prime} \phi\right)^{\prime} \phi \leqq\left((e \phi)^{\prime}\right)^{\prime}=e \phi$. Clearly $e \leqq\left((e \phi)^{\prime} \phi\right)^{\prime}$. Using monotony, we get from the latter inequality $e \phi \leqq\left((e \phi)^{\prime} \phi\right)^{\prime} \phi$. Hence $e \phi=\left((e \phi)^{\prime} \phi\right)^{\prime} \phi$.

RemarK 1. Let $L$ be an orthomodular lattice. A projection $\phi \in P(L)$ is a join-homomorphism of $L$ [2, Theorem 5.2, page 37]. On the other hand every join-homomorphism is monotone. From $(1 \phi)^{\prime} \phi \leqq 1^{\prime}$ we get $0 \phi=0$, where $0:=1^{\prime}$. Therefore $P(L)$ coincides with the set of projections introduced by Foulis [4], namely the set of idempotent, self-adjoint hemimorphisms on $L$.

One verifies that in an involution poset $T$ a closure operator $\gamma$ satisfies

$$
\left((e \gamma)^{\prime} \gamma\right)^{\prime} \gamma \leqq e \gamma \quad(e \in T)
$$

Those closure operators for which the equality

$$
\left((e \gamma)^{\prime} \gamma\right)^{\prime} \gamma=e \gamma \quad(e \in T)
$$

is valid are of special interest. As we will see below they are closely related to projections and determining for the lattice and orthomodular structure of $T$. We call these operators c-closure operators and denote with $C(T)$ the set of all $c$-closure operators on an involution poset $T$. The mappings $I$ and $e J:=1$ are $c$-closure operators.

$C(T)$ is partially ordered by means of the ordering relation

$$
\gamma_{1} \leqq \gamma_{2}: \Leftrightarrow e \gamma_{2} \leqq e \gamma_{1} \quad(e \in T) .
$$

$I$ is the largest and $J$ the smallest element of $C(T)$.

THEOREM 2. Let $T$ be an involution poset. If $\gamma$ is a c-closure operator, then $\left((e \gamma)^{\prime} \gamma\right)^{\prime}$ is a projection on $T$. If $\phi$ is a projection, then $\left((e \phi)^{\prime} \phi\right)^{\prime}$ is a c-closure operator on $T$.

The mapping $\gamma \in C(T) \rightarrow \phi \in P(T)$ where $e \phi:=\left((e \gamma)^{\prime} \gamma\right)^{\prime}$ is one-to-one and maps the set of c-closure operators onto the set of projections on T. $\phi \in P(T) \rightarrow$ $\gamma \in C(T)$ where $e \gamma:=\left((e \phi)^{\prime} \phi\right)^{\prime}$ is the corresponding inverse mapping.

PRoOF. Clearly the mapping $e \rightarrow\left((e \gamma)^{\prime} \gamma\right)^{\prime}$ is monotone. Using properties of $c$-closure operators, we get

$$
\left(\left(\left((e \gamma)^{\prime} \gamma\right)^{\prime} \gamma\right)^{\prime} \gamma\right)^{\prime}=\left((e \gamma)^{\prime} \gamma\right)^{\prime},
$$

which proves idempotence of the mapping. Furthermore 


$$
\left(\left(\left(\left((e \gamma)^{\prime} \gamma\right)^{\prime}\right)^{\prime} \gamma\right)^{\prime} \gamma\right)^{\prime}=\left(\left((e \gamma)^{\prime} \gamma\right)^{\prime} \gamma\right)^{\prime}=(e \gamma)^{\prime} \leqq e^{\prime} .
$$

Hence the mapping is a projection.

Let $\phi$ be a projection. By i) and iii) of the definition of a projection, one easily sees that the mapping $e \gamma:=\left((e \phi)^{\prime} \phi\right)^{\prime}$ is monotone and majorizes the argument. By Lemma 1 and the basic properties of projections we get

$$
(e \gamma) \gamma=\left(\left(\left((e \phi)^{\prime} \phi\right)^{\prime} \phi\right)^{\prime} \phi\right)^{\prime}=\left((e \phi)^{\prime} \phi\right)^{\prime}=e \gamma
$$

and

$$
\left((e \gamma)^{\prime} \gamma\right)^{\prime} \gamma=\left(\left(\left(\left(\left((e \phi)^{\prime} \phi\right) \phi\right)^{\prime} \phi\right) \phi\right)^{\prime} \phi\right)^{\prime}=\left(\left(\left((e \phi)^{\prime} \phi\right)^{\prime} \phi\right)^{\prime} \phi\right)^{\prime}=\left((e \phi)^{\prime} \phi\right)^{\prime}=e \gamma .
$$

Hence $\gamma \in C(T)$.

For all $\phi \in P(T), \gamma \in C(T)$ and $e \in T$

$$
\left(\left(\left((e \gamma)^{\prime} \gamma\right)^{\prime \prime} \gamma\right)^{\prime} \gamma\right)^{\prime \prime}=\left((e \gamma)^{\prime} \gamma\right)^{\prime} \gamma=e \gamma
$$

and

$$
\left(\left(\left((e \phi)^{\prime} \phi\right)^{\prime \prime} \phi\right)^{\prime} \phi\right)^{\prime \prime}=\left((e \phi)^{\prime} \phi\right)^{\prime} \phi=e \phi
$$

is valid. This proves the second part of the theorem.

Remark 2. Because of the one-to-one correspondence between $P(T)$ and $C(T)$ the ordering in the set of $c$-closure operators induces an ordering in the set of projections as follows:

Let $\phi_{1}, \phi_{2}$ be two projections and $\gamma_{1}, \gamma_{2}$ the corresponding $c$-closure operators. The relation

$$
\phi_{1} \leqq \phi_{2}: \Leftrightarrow \gamma_{1} \leqq \gamma_{2}
$$

is an ordering relation that makes $P(T)$ into a partially ordered set. The mapping $\gamma \rightarrow \phi$ where $e \phi:=\left((e \gamma)^{\prime} \gamma\right)^{\prime}$ can then be interpreted as an order-isomorphism between the posets $C(T)$ and $P(T)$.

The next two lemmata lead us to the main result of this paper.

Lemma 3. Let $T$ be an orthocomplemented poset and $\gamma \in C(T)$. Then

i) $e \gamma \vee(e \gamma)^{\prime} \gamma$ exists and is equal to 1 ,

ii) $e \gamma \wedge(e \gamma)^{\prime} \gamma$ exists and is equal to $0 \gamma$.

Proof. i) Of course $e \gamma \leqq 1$ and $(e \gamma)^{\prime} \gamma \leqq 1$. If there is an $f \in T$ such that $e \gamma \leqq f$ and $(e \gamma)^{\prime} \gamma \leqq f$, then also $(e \gamma)^{\prime} \leqq f$ since $(e \gamma)^{\prime} \leqq(e \gamma)^{\prime} \gamma$. But $e \gamma \vee(e \gamma)^{\prime}=1$, hence $1 \leqq f$. This proves that $e \gamma \vee(e \gamma)^{\prime} \gamma=1$. ii) By monotony $o \gamma \leqq e \gamma$ and $0 \gamma \leqq(e \gamma)^{\prime} \gamma$. Let $f \in T$ be an element such that $f \leqq e \gamma$ and $f \leqq(e \gamma)^{\prime} \gamma$. By monotony and idempotence of the closure operator we get

$$
f \gamma \leqq e \gamma \text { and } f \gamma \leqq\left(e \gamma^{\prime}\right) \gamma \text { or }(e \gamma)^{\prime} \leqq(f \gamma)^{\prime}
$$


and $\left((e \gamma)^{\prime} \gamma\right)^{\prime} \leqq(f \gamma)^{\prime}$. Again by monotony we have then $(e \gamma)^{\prime} \gamma \leqq(f \gamma)^{\prime} \gamma$ and

$$
e \gamma=\left((e \gamma)^{\prime} \gamma\right)^{\prime} \gamma \leqq(f \gamma)^{\prime} \gamma .
$$

According to part i) of this proof, this implies that $(f \gamma)^{\prime} \gamma=1$ or $\left((f \gamma)^{\prime} \gamma\right)^{\prime}=0$. Finally we get $f \leqq f \gamma=\left((f \gamma)^{\prime} \gamma\right)^{\prime} \gamma=0 \gamma$. Thus $e \gamma \wedge(e \gamma)^{\prime} \gamma=0 \gamma$.

LEMMA 4. Let $T$ be an involution poset and $\gamma$ a c-closure operator, then $(0 \gamma)^{\prime} \gamma=1$.

Proor. By theorem 2 there is a projection $\phi$ such that $e \gamma=\left((e \phi)^{\prime} \phi\right)^{\prime}$. Since $0 \phi=0$ and by lemma 1 we get

$$
(0 \gamma)^{\prime} \gamma=\left(\left(\left((0 \phi)^{\prime} \phi\right)^{\prime \prime} \phi\right)^{\prime} \phi\right)^{\prime}=\left(\left((0 \phi)^{\prime} \phi\right)^{\prime} \phi\right)^{\prime}=(0 \phi)^{\prime}=1 .
$$

THEOREM 5. Let $T$ be an involution poset. $T$ is an orthomodular lattice if and only if every interval $[e, 1](e \in T)$ is the range of a c-closure operator.

Proof. Assume that $T$ is an orthomodular lattice. One verifies that for a given interval $[e, 1]$ the mapping $f \rightarrow e \vee f$ is a closure operator that maps $T$ onto it. We show that this mapping has the characteristic property of $c$-closure operators.

Since $e \leqq e \vee f$, there exists by orthomodularity of the lattice $T$ an element $g \in T$ such that $e \vee g=e \vee f$ and $e \leqq g^{\prime}$. Now

$e \vee\left(e \vee(e \vee f)^{\prime}\right)^{\prime}=e \vee\left(e \vee(e \vee g)^{\prime}\right)^{\prime}=e \vee\left(e^{\prime} \wedge(e \vee g)\right)=e \bigvee\left(e^{\prime} \wedge g\right)=e \vee g=e \vee f$

Conversely, we prove first that $T$ must be a lattice. When $e, f \in T$, then there is a $c$-closure operator $\gamma$ that maps $T$ onto the interval $[f, 1]$. Clearly $e \leqq e \gamma$ and $f=0 \gamma \leqq e \gamma$. Let $g \in T$ be an element such that $e \leqq g$ and $f \leqq g$. Since $\gamma$ maps $T$ onto $[f, 1]$, it follows fron the latter inequality that $g \gamma=g$. From $e \leqq g$ we then get $e \gamma \leqq g \gamma=g$. Thus $e \vee f$ exists in $T$ and is equal to $e \gamma$.

Let $\gamma \in C(T)$ with $T \gamma=[e, 1]$. By lemma 4 we get $1=(0 \gamma)^{\prime} \gamma=e^{\prime} \gamma=e^{\prime} \vee e$ for all $e \in T$. Therefore $T$ is an orthocomplemented lattice.

Now we prove orthomodularity of the lattice $T$. Let $e \leqq f$ and $y \in C(T)$ such that $T \gamma=[e, 1]$. We again have $e=0 \gamma$ and $f \gamma=f$. By Lemma 3 (ii) and the result above we get $e=0 \gamma=f \gamma \wedge(f \gamma)^{\prime} \gamma=f \wedge f^{\prime} \gamma=f \wedge\left(e \vee f^{\prime}\right)$.

Remark 3. Let $L$ be an orthomodular lattice. By Theorem 2 and Remark 1 the mappings $e \rightarrow e \phi:=\left((e \gamma)^{\prime} \gamma\right)^{\prime}(y \in C(L))$ are the projections in the Baer *-semigroup of hemimorphisms on $L$. One can prove that

$$
\left(e \phi_{1}\right) \phi_{2}=e \phi_{1}\left(\phi_{1}, \phi_{2} \in P(L) ; e \in L\right) \Leftrightarrow \phi_{1} \leqq \phi_{2},
$$

thus the usual ordering of projections coincides with that induced by the poset $C(L)$ (Remark 2). The closed projections, namely the Sasaki-projections, are giver by $\left(\left(e \gamma_{f}\right)^{\prime} \gamma_{f}\right)^{\prime}(f \in L)$ where $\gamma_{f} \in C(L)$ and $L \gamma_{f}=[f, 1]$. 
Note that a mapping $\gamma$ is a symmetric closure operator on $L[5]$ if and only if $\gamma$ is a $c$-closure operator for which $0 \gamma=0$ is valid. Furthermore, the symmetric closure operators are the fixelements of the mappings exhibited in theorem 2.

\title{
References
}

[1] G. Birkhoff, Lattice Theory, (Amer. Math. Soc., 3rd ed. (1967)).

[2] T. S. Blyth and M. F. Janowitz, Residuation Theory, (Pergamon Press (1972)).

[3] P. D. Finch, 'Sasaki-Projections on Orthoçomplemented Posets', Bull. Austral. Math. Soc. 1 (1969), 319-324.

[4] D. J. Foulis, 'Baer *-semigroups', Proc. Amer. Math. Soc. 11 (1960), 648-654.

[5] M. F. Janowitz, 'Residuated Closure Operators', Portugal. Math. 26 (1967), 221-252.

\author{
University of Calgary \\ Calgary \\ Alberta, Canada \\ University of Berne \\ Theoretical Physics \\ Berne, Switzerland
}

